\title{
Chlamydophila pneumoniae Infection Induces Alterations in Vascular Contractile Responses
}

\author{
Justin F. Deniset, Thomas E. Hedley, \\ Elena Dibrov, and Grant N. Pierce \\ From the Institute of Cardiovascular Sciences, St. Boniface \\ Hospital Research Centre, Department of Physiology, Faculties of \\ Medicine and Pharmacy, University of Manitoba, Winnipeg, \\ Manitoba, Canada
}

Cblamydophila pneumoniae infection has been associated in previous studies with coronary artery disease. The live bacterium has been detected within atherosclerotic plaques and can induce the structural remodeling of the vessel wall. However, the direct effects of infection on the contractile characteristics of the arteries remain unknown. Left anterior descending coronary arteries isolated from porcine hearts were dissected and placed in culture medium for 72 hours before infection with $C$. pneumoniae. Contractile responses to high molar $\mathrm{KCl}$ and $\mathrm{u} 46619$ levels and relaxation responses to bradykinin and sodium nitroprusside were assessed at days 5 and $\mathbf{1 0}$ postinfection. C. pneumoniae induced decreases in both KCl- and u46619-induced contractile responses at both time points. The altered contractile responses coincided with a down-regulation of L-type $\mathrm{Ca}^{2+}$ channels at both time points and inositol 1,4,5-triphosphate receptor (IP3R) levels at day 10 postinfection. Infection also induced attenuation of the endothelial-dependent relaxation response to bradykinin at day 10 postinfection. A decrease in endothelial nitric oxide synthase expression levels was noted at day 10 postinfection. Furthermore, an increase in superoxide production combined with an increase in p22phox expression levels was also observed at this time point. These findings indicate that $C$. pneumoniae infection can directly alter the vascular contractile responses in porcine coronary arteries, providing additional evidence for the role of $C$. pneumoniae infection in cardiovascular disease. (Am J Pathol 2012, 180:1264-1272; DOI: 10.1016/j.ajpath.2011.11.005)

Chlamydophila pneumoniae (C. pneumoniae), a common respiratory pathogen, has been associated with the inci- dence of cardiovascular disease. ${ }^{1-4}$ Live C. pneumoniae can be detected in atherosclerotic carotid and coronary arteries, $^{5-8}$ and growth of the bacterium is supported by endothelial cells (ECs) and vascular smooth muscle cells (VSMCs). ${ }^{9-11}$ Endothelial dysfunction and VSMC proliferation are among the proposed mechanisms for the involvement of $C$. pneumoniae in the development of atherosclerosis. These manifestations have potential consequences for the vascular contractile characteristics of infected vasculature.

Endothelial dysfunction, a key initiating step in the progression of atherosclerosis, results primarily from a reduced bioavailability of nitric oxide (NO) leading to impaired endothelial-dependent relaxation. ${ }^{12}$ Infectious burden has been noted as an independent risk factor for endothelial dysfunction. ${ }^{13}$ Chronic and acute inoculations with the $C$. pneumoniae have been demonstrated to induce endothelial dysfunction in mice and pigs respectively. ${ }^{14-17}$ However, it is difficult to establish a causative role for $C$. pneumoniae in endothelial dysfunction with an in vivo system because of the involvement of an active immune response to infection. In vitro findings have provided some evidence for a direct role of the bacterium in promoting this dysfunctional state. C. pneumoniae infection can directly decrease endothelial nitric oxide synthase expression (eNOS), the primary source of $\mathrm{NO}$ within the vessel wall. ${ }^{18}$ Chlamydial heat shock protein 60 (cHSP60), produced during active chlamydial replication, induces a decrease in eNOS expression and activity in ECs and stimulates endothelial dysfunction in isolated porcine coronary arteries. ${ }^{19}$ Endogenous HSP60, which is up-regulated in response to $C$. pneumoniae infection, ${ }^{20,21}$ promotes endothelial activation and is associated with endothelial dysfunction in adolescents. ${ }^{22,23}$

Supported by a grant from the Canadian Institutes for Health Research $(\mathrm{ClHR})$ and with indirect funding from the St. Boniface Hospital \& Research Foundation. J.D. is the recipient of a CIHR Frederick Banting and Charles Best Doctoral Canada Graduate Scholarship.

Accepted for publication November 11, 2011.

CME Disclosure: None of the authors disclosed any relevant financial relationships.

Address reprint requests to Grant N. Pierce, Ph.D., Institute of Cardiovascular Sciences, St. Boniface Hospital Research Centre, 351 Taché Ave, Winnipeg, MB, Canada R2H 2A6. E-mail: gpierce@sbrc.ca. 
Whether C. pneumoniae infection can induce similar cellular changes within a complex vascular environment leading to endothelial dysfunction in the absence of an immune response is thus worth investigating.

VSMC proliferation, another key feature of atherosclerotic development, also could have important implications for the functional characteristics of arterial vessels. During the proliferative process, VSMCs undergo a phenotypic switch from a contractile (differentiated) VSMC to a synthetic (de-differentiated) VSMC. This de-differentiation process typically involves alterations in the expression of contractile proteins such as smooth muscle $\alpha$ actin and smooth muscle myosin heavy chain as well as $\mathrm{Ca}^{2+}$ handling proteins like the $\mathrm{L}$-type $\mathrm{Ca}^{2+}$ channel. ${ }^{24}$ As a result, the synthetic phenotype observed in proliferative VSMC could severely alter a vessel's function and has been suggested to play an important pathophysiological role in the development of vascular diseases such as atherosclerosis, restenosis and hypertension. ${ }^{25}$ Direct C. pneumoniae infection and treatment with cHSP60 can induce VSMCs proliferation. ${ }^{21,26,27}$ However, little is known about how $C$. pneumoniae-mediated proliferation can influence vascular function responses within a complete vessel system.

The current investigation aims to determine the direct action of $C$. pneumoniae infection on the functional responses of coronary arteries. We have recently developed an ex vivo porcine coronary artery model that allows us to determine the direct effect of $C$. pneumoniae infection within an arterial environment in the absence of an immune response. ${ }^{27}$ Using this approach, C. pneumoniae was able to infect the endothelial layer, migrate into the medial layer, and actively replicate within the vessel wall for up to 10 days postinfection. This active replication resulted in significant arterial thickening through induction of vascular smooth muscle cell (VSMC) proliferation. This current study uses this ex vivo coronary artery model to investigate vascular contractile and relaxation responses within C. pneumoniae-infected vessels before and after vascular hypertrophic responses.

\section{Materials and Methods}

\section{Reagents and Antibodies}

Cycloheximide, bradykinin acetate, 9,11-Dideoxy-11 $\alpha$, $9 \alpha$-epoxy-methanoprostaglandin $F_{2 \alpha}$ (u46619), sodium nitroprusside, anti-smooth muscle myosin, and antismooth muscle actin antibodies were obtained from Sigma-Aldrich (St. Louis, MO). The anti-IP3 receptor-1 and anti-L-type of voltage-gated $\mathrm{Ca}^{2+}$ channel antibodies were obtained from Alomone Labs (Jerusalem, Israel). The antieNOS antibody was obtained from BD Transduction Laboratories (Franklin Lakes, NJ). The anti-iNOS antibody was obtained from Abcam (Cambridge, MA). The antip22phox antibody was obtained from Santa Cruz (Santa Cruz, CA). The I $\mathrm{B}_{\mathrm{B}}-\alpha$ antibody was obtained from Cell Signaling (Danvers, MA). The chlamydial HSP60 antibody was obtained from Affinity BioReagents (Golden, CO). The human HSP60 antibody was obtained from
Stressgen (Ann Arbor, MI). The anti-NF $\kappa$ B antibody, HRPconjugated anti-mouse IgG and HRP-conjugated anti-rabbit IgG antibodies were obtained from Millipore (Billerica, MA). The dihydroethidium, Alexa Fluor 488-conjugated anti-mouse IgG and Alexa Fluor 488-conjugated antirabbit IgG antibodies were obtained from Invitrogen (Carlsbad, CA).

\section{Propagation of C. pneumoniae}

C. pneumoniae AR39 strain was obtained from the University of Washington (Seattle, WA). The organism was propagated in HL cells as described previously. ${ }^{28}$ The purified organism was resuspended in chlamydial sucrose-phosphate-glutamate medium (SPG) and stored at $-80^{\circ} \mathrm{C}$ until use. The titer of $\mathrm{C}$. pneumoniae was determined in cycloheximide-treated HL cells, and concentrations used were expressed as inclusion-forming units (IFU) per milliliter. ${ }^{29}$

\section{Isolation and Infection of Porcine Coronary Arteries}

Coronary artery isolation, culturing, and C. pneumoniae infection were performed as previously described. ${ }^{27}$ SPG and heat-inactivated C. pneumoniae (mock infection) were used for treatment and infection controls, respectively. After infection, vessels were maintained in culture for up to 10 days. Organ culture medium was changed (without antibiotics) every 48 hours, and the old medium was collected for conditioned medium experiments and protein analysis. C. pneumoniae infection induced arterial thickening in this model starting at day 6 postinfection. To determine whether alterations in vascular response preceded or followed hypertrophy, parameters were examined at two main time points, days 5 and 10 postinfection.

\section{Vascular Function Assessment}

Vascular function was assessed for coronary segments collected at days 4 and 5 and days 9 and 10 postinfection, using the experimental protocol previously described. ${ }^{27}$ All functional experiments were completed within a laminar flow biosafety cabinet to prevent spread of infection.

\section{Treatment of Human Umbilical Vein Endothelial Cells with Conditioned Medium}

Human umbilical vein endothelial cells (HUVECs) were obtained from Lonza and maintained in culture conditions with EGM-2 (Lonza, Walkersville, MD). Cells from P3 to P6 were used for experiments. HUVECS were plated at $2 \times 10^{6}$ cells per well in a 6 -well plate. Organ culture medium was collected every 48 hours during media changes. Media from similar treatments were combined in pairs; a portion (2 $\mathrm{mL}$ ) of the medium was placed directly over HUVECS plated in 6-well plates. HUVECS were collected at 24 hours after incubation 
with conditioned medium. The additional medium (1 $\mathrm{mL}$ ) was collected and spun at $1105 \mathrm{rpm}$ for 5 minutes. The supernatant was collected and stored at $-80^{\circ} \mathrm{C}$ for protein analysis.

\section{Western Blot Analysis}

To assess protein expression levels within treated vessels, coronary segments were collected at days 4 and 5 and days 9 and 10 postinfection. On collection, segments were rinsed with PBS, flash-frozen in liquid nitrogen and stored at $-80^{\circ} \mathrm{C}$. Coronary segments were subsequently ground and re-suspended in RIPA buffer (50 mmol/L Tris- $\mathrm{HCl}, \mathrm{pH} 7.5,150 \mathrm{mmol} / \mathrm{L} \mathrm{NaCl}, 1$ $\mathrm{mmol} / \mathrm{L}$ EDTA, $1 \mathrm{mmol} / \mathrm{L}$ EGTA, $1 \%$ Triton $100,0.1 \%$ SDS, $0.5 \%$ sodium deoxycholate, $1 \mu \mathrm{g} / \mathrm{mL}$ leupeptin, 1 $\mathrm{mmol} / \mathrm{L}$ PMSF, $1 \mathrm{mmol} / \mathrm{L}$ protease inhibitor cocktail, 1 $\mathrm{mmol} / \mathrm{L}$ DTT, and $1 \mathrm{mmol} / \mathrm{L}$ benzimidine). HUVECs collected after conditioned medium treatment were resuspended in RIPA buffer, sonicated, spun at 14,000 rpm for 10 minutes at $4{ }^{\circ} \mathrm{C}$, and the supernatant was collected for Western blotting. Protein assays were performed to determine concentrations within tissue and HUVEC lysates. Lanes were loaded with $5 \mu \mathrm{g}$ of tissue lysate for detection of smooth muscle actin and myosin and $30 \mu \mathrm{g}$ of protein for the other targeted proteins. Tissue samples were run in duplicate, with half of the blot used to probe with antibodies and the other half used for amido black staining. For HUVEC lysates, lanes were loaded with $30 \mu \mathrm{g}$ of protein. Proteins were separated using a $4 \%$ to $12 \%$ gradient denaturing polyacrylamide gel and transferred electrophoretically onto a nitrocellulose membrane. Membranes were incubated with anti-smooth muscle actin $(1: 20,000)$, anti-smooth muscle myosin $(1: 1000)$, anti-IP3 receptor-1(1:500), anti-L-type of voltage-gated $\mathrm{Ca}^{2+}$ channel (1:500), anti-eNOS (1:200), anti-iNOS (1:500), anti-p22phox (1:500), anti-NF $\kappa$ B (1:1000), antiI $\mathrm{KB}(1: 1000)$, or anti-GAPDH (1:5000) antibodies. HRPconjugated anti-mouse $\lg G$ and anti-rabbit IgG were used as secondary antibodies. Bands were visualized with the Supersignal West Pico or Femto Chemiluminescent Substrates (Pierce, Rockford, IL). Bands and Amido Black staining were quantified by densitometry using Quantity One software (Bio-Rad, Hercules, CA). Protein expression for tissue and HUVEC lysates were normalized by Amido Black staining and GAPDH, respectively.

Protein concentrations of $\mathrm{CHSP} 60$ and hHSP60 within the conditioned medium were also assessed using Western blot methodology. A trichloroacetic acid precipitation protocol was performed to concentrate protein in conditioned medium. In short, $250 \mu \mathrm{l}$ of the conditioned medium was combined with half volume of $50 \%$ trichloroacetic acid; the precipitate was spun down, washed with acetone, and spun; and the pellet was reconstituted in 40 $\mu \mathrm{l}$ of $0.5 \mathrm{~mol} / \mathrm{L}$ of $\mathrm{NaOH}$ and $40 \mu \mathrm{l}$ of $2 x$ SDS-PAGE loading buffer. A $40-\mu$ l quantity of the concentrated protein lysate from each treatment at days $4,6,8$, and 10 postinfection were loaded along with varying amounts of recombinant cHSP60 or hHSP60 (1 to $0.01 \mu \mathrm{g}$ of protein) used to construct a standard curve. Proteins were separated using a $9 \%$ gradient denaturing polyacrylamide gel and transferred electrophoretically onto a nitrocellulose membrane. Membranes were incubated with either anticHSP60 (1:1000) or anti-hHSP60 (1:1000) antibodies, and HRP-conjugated anti-mouse IgG was used as secondary antibody. Bands were visualized with the Supersignal West Pico or Femto Chemiluminescent Substrates (Pierce). Bands and Amido Black staining were quantified by densitometry with Quantity One software (Bio-Rad).

\section{In Situ Superoxide Detection and Immunofluorescence Staining}

Coronary segments were collected at days 9 and 10 postinfection, fixed in ethanol, rehydrated, and subsequently equilibrated in 30\% sucrose solution before mounting in OCT compound (Tissue-tek, Sakura Finetek, Torrance, CA). These OCT mounted segments were slowly frozen to $-80^{\circ} \mathrm{C}$, cut (HM 500 OM Cryostat, Microm) into $8-\mu \mathrm{m}$ sections and placed on positively coated glass slides. To evaluate protein expression in coronary sections, antigen retrieval was first performed as previously described. ${ }^{27}$ Subsequently, sections were incubated with anti-eNOS(1:25), anti-p22phox (1:50), antiTLR4 (1:10), anti-cHSP60 (1:50) and anti-hSP60 antibodies. Texas Red-conjugated anti-mouse IgG (1: 750), Alexa Fluor 488-conjugated anti-mouse IgG (1: 1000 ), and Alexa Fluor 488-conjugated anti-rabbit IgG $(1: 1000)$ were used as secondary antibodies for the respective primary antibodies. Hoescht staining solution (5 $\mathrm{ng} / \mathrm{mL}$ ) was added to the slides to identify nuclei. To determine the levels of superoxide anions within the vessels, sections were incubated with dihydroethidium as previously described. ${ }^{30}$ Slides were fixed with FluoroSave reagent (Calbiochem, San Diego, CA) to preserve fluorescence. Staining was visualized using a fluorescent inverted microscope (TE-2000s; Nikon) and mean intensity was quantified using NIS Elements imaging software (Nikon, Mississauga, ON).

\section{Statistical Analysis}

Data are presented as mean \pm SEM unless otherwise stated. Differences between treatment groups were assessed by one-way analysis of variance using the Student-Newman-Keuls method. A probability of $P<0.05$ was considered statistically significant.

\section{Results}

\section{C. pneumoniae Induces Alterations in Vascular Contractile Function}

Isolated coronary vessels infected with C. pneumoniae displayed significant decreases in maximal contractile response to $\mathrm{KCl}$ when compared with control and mockinfected vessels at both days 5 (Figure $1 \mathrm{~A}$ ) and 10 postinfection (Figure 1B). Similar qualitative responses were 

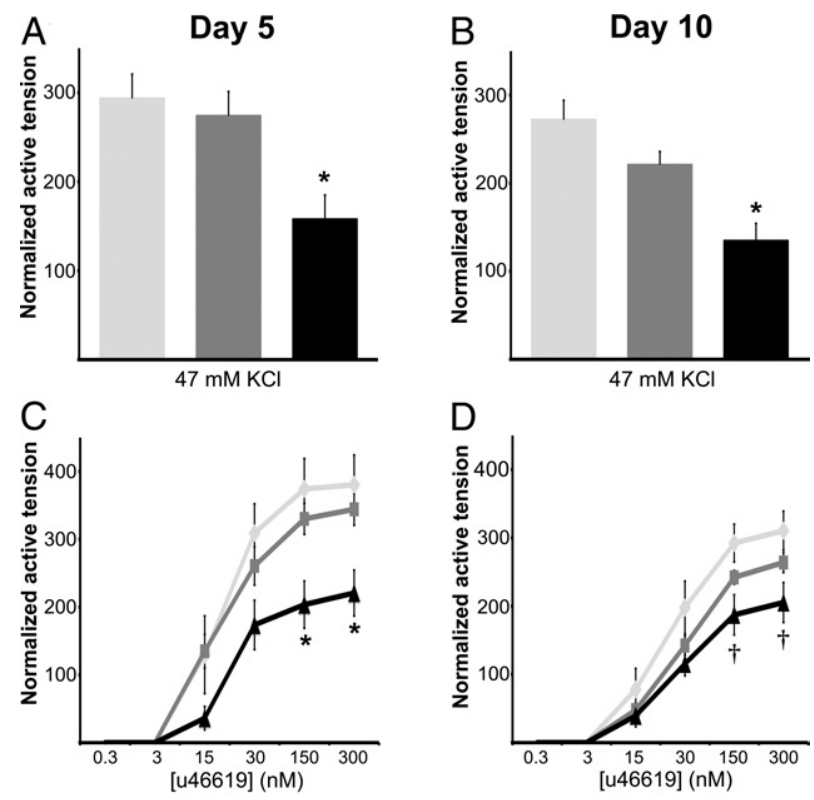

Control

Mock Infection

Figure 1. Contractile responses of infected isolated vascular rings. Maximal contractile response to $47 \mathrm{mmol} / \mathrm{L} \mathrm{KCl}$ day 5 postinfection (A) and day 10 postinfection (B) and 0.3 to $300 \mathrm{nmol} / \mathrm{L} \mathrm{u} 46619$ day 5 postinfection $(\mathbf{C})$ and day 10 postinfection $(\mathbf{D})$. All values are mean \pm SEM, $n=7$ to 11 . ${ }^{*} P<0.05$ versus control and mock infection; ${ }^{\dagger} P<0.05$ versus control.

found in response to receptor-mediated contraction with u46619 (Figure 1, C and D). At day 5 postinfection, the contractile response to both 150 and $300 \mathrm{nmol} / \mathrm{L} \mathrm{u} 46619$ in C. pneumoniae-infected segments was decreased compared with control and mock-infected vessels. At day 10 postinfection, the contractile response to u46619 in infected segments was decreased only when compared with control.

\section{C. pneumoniae Infection Alters Expression of Calcium-Handling Proteins but Not Contractile Proteins}

Expression of the smooth muscle contractile proteins actin and myosin heavy chain was assessed in vessels at days 5 and 10 postinfection (Figure 2, A and B). Expression levels appeared to decrease with $C$. pneumoniae infection; however these changes were not significantly different compared with other treatment at day 5 (smooth muscle actin, $P=0.087$; myosin, $P=0.172$ ) and day 10 (smooth muscle actin, $P=0.145$; myosin, $P=0.143$ ). The expression of calcium handling proteins involved in smooth muscle contraction, L-type voltage gated $\mathrm{Ca}^{2+}$ channel and $I P_{3}$ receptor, was also determined at days 5 and 10 postinfection (Figure 3, A and B). The expression of the L-type $\mathrm{Ca}^{2+}$ channel was significantly decreased in C. pneumoniae-infected vessels compared with control and mock-infected vessels at day 5 postinfection (Figure $3 A$ ) and day 10 (Figure 3B). The expression of $I P_{3}$ receptor was unchanged between groups at day 5 postinfection (Figure 3C) but was significantly decreased in infected vessels compared with control at day 10 postinfection (Figure 3D).

\section{C. pneumoniae Infection Attenuates Endothelial Dependent Relaxation in Isolated Coronary Vessels}

Precontraction levels in response to $30 \mathrm{nmol} / \mathrm{L}$ u46619 remained unchanged between treatment groups at both days 5 and 10 postinfection (data not shown). Endothelial-dependent relaxation responses to bradykinin remained unaltered between groups at day 5 postinfection (Figure 4A), but at day 10 (Figure 4B) were significantly reduced in infected vessels compared with control at $10^{-9}$ to $10^{-6} \mathrm{~mol} / \mathrm{L}$ and at $10^{-7}$ to $10^{-6} \mathrm{~mol} / \mathrm{L}$ in comparison to mock-infected vessels (Figure 4B). No change in endothelial-independent relaxation responses to sodium nitroprusside was observed among groups at either time point (Figure 4, C and D).

\section{C. pneumoniae Infection Induces Decrease in eNOS Expression}

Expression of eNOS and iNOS, the nitric oxide producing enzymes within the vessel was determined via Western blot analysis. eNOS expression was unchanged among treatment groups at day 5 postinfection (Figure 5A). At day 10 postinfection, eNOS was down-regulated in infected vessels compared with control and mock-infected vessels (Figure 5B). Further analysis via immunostaining of coronary vessels at day 10 postinfection revealed a significant decrease in the mean fluorescence of infected
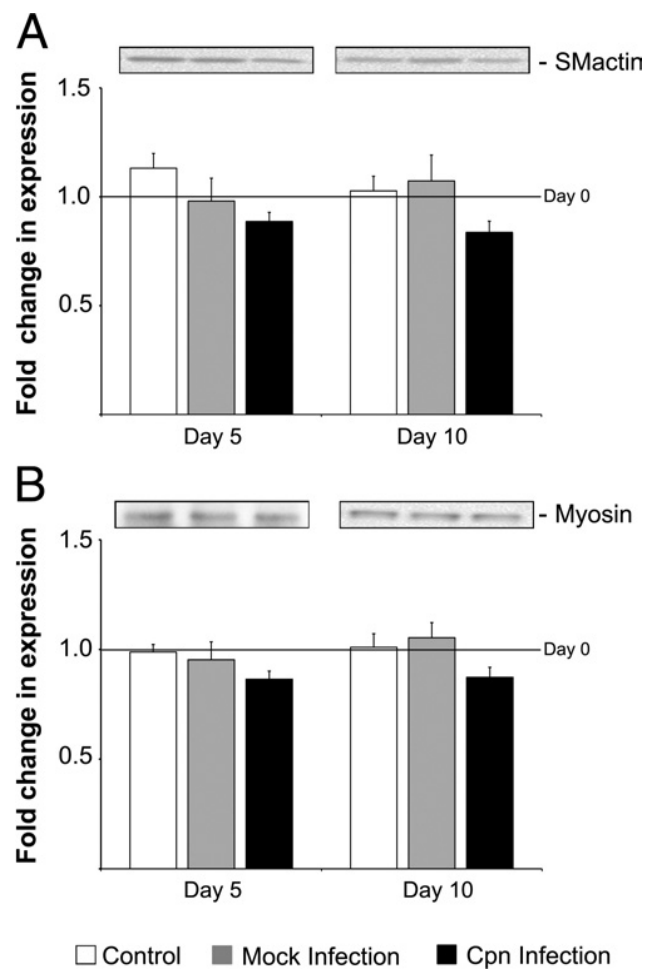

Figure 2. Western blot analysis of contractile proteins. Smooth muscle actin (A) and smooth muscle myosin (B) expression at day 5 and day 10 postinfection in infected coronary segments. Representative Western blots are displayed above their corresponding graphs. Expression levels are represented as fold change over day 0 tissue \pm SEM, $n=6$. 

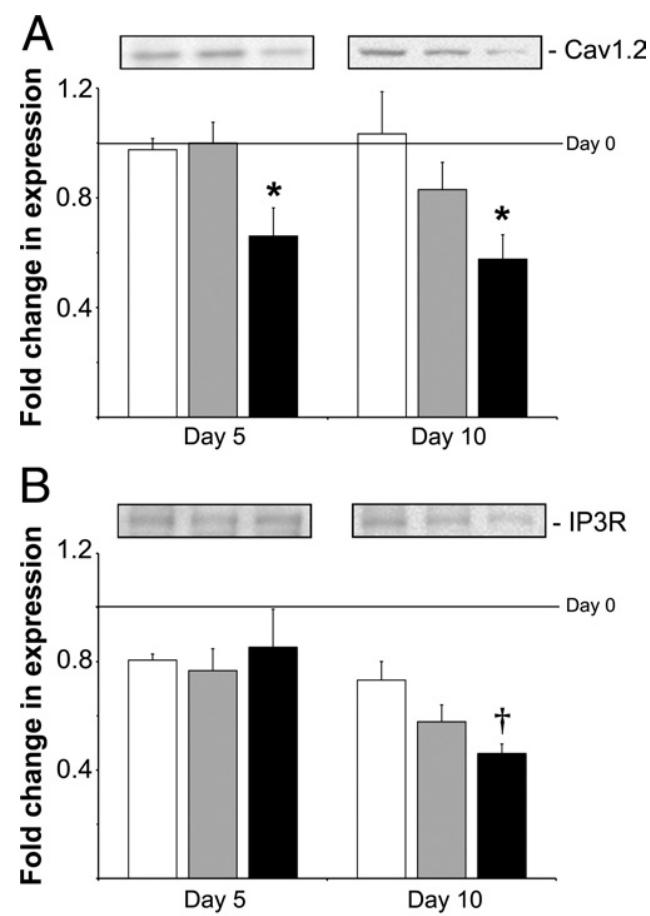

\section{$\square$ Control $\square$ Mock Infection $\square$ Cpn Infection}

Figure 3. Western blot analysis of $\mathrm{Ca}^{2+}$ handling proteins. L-type $\mathrm{Ca}^{2+}$ channel (Cav1.2) (A) and IP3R expression (B) at day 5 and day 10 postinfection in infected coronary segments. Representative Western blots are displayed above their corresponding graphs. Expression levels are represented as fold change over day 0 tissue \pm SEM, $n=4$ to $6 .{ }^{*} P<0.05$ versus control and mock infection; ${ }^{\dagger} P<0.05$ versus control.

cross-sections compared with control and mock-infected tissues (Figure 5C). iNOS expression remained unchanged between treatment groups at both time points (data not shown).

\section{C. pneumoniae Components Stimulates Superoxide Anion Production and p22phox Expression}

Expression of p22phox, a major subunit of $\mathrm{NADPH}$ oxidase, was determined via Western blot (Figure 6A) and immunostaining (Figure 6, B and C). Expression of p22phox, as detected by Western blots, was not changed among groups at day 5 postinfection but was up-regulated in both mock-infected and C. pneumoniaeinfected vessels compared with control vessels at day 10 postinfection (Figure 6, A and B). p22phox immunostaining at day 10 postinfection confirmed these results, with higher fluorescence levels in mock-infected and C. pneumoniae-infected vessels compared with control (Figure 6C).

Superoxide anion production at day 10 postinfection was visualized using dihydroethidium staining on infected coronary cross-sections (Figure 7). Quantification of this staining revealed significant increases in mean fluorescence in both mock-infected and C. pneumoniaeinfected vessels compared with control $(a$ 99\% $(P<0.05)$ and $207 \%(P<0.01)$ change in comparison to control, respectively.

\section{$N F_{\kappa} B$ Activation Is Not Altered in Infected Coronary Arteries}

The NF $\kappa$ B pathway is known to be involved in the inflammatory process within the vessel wall, as such both $\mathrm{NF} \kappa \mathrm{B}$ and $I_{\kappa} \mathrm{B}$ protein expression was assessed. Expression of both proteins did not significantly change between treatments at days 5 and 10 postinfection. The ratios of $I_{\kappa} \mathrm{B}$ to $\mathrm{NF} \kappa \mathrm{B}$ in both $\mathrm{C}$. pneumoniae-infected and mock-infected vessels varied from control vessels but not enough to be statiscally significant.

\section{C. pneumoniae Increases TLR4, hHSP60, and cHSP60 Expression in the Endothelial Layer}

TLR4, cHSP60, and hHSP60 localization along the endothelial layer was determined via immunostaining (Figure 8). Both C. pneumoniae-infected and mockinfected vessels displayed an increase in TLR4 and hHSP60 expression when compared with nontreated vessels (Figure $8, D$ and $E$ compared with $G$ and $H$ ). In addition, CHSP60 expression could be localized along the endothelial layer in C. pneumoniae-infected vessels (Figure 8I) but was absent in both control and mockinfected coronary vessels.
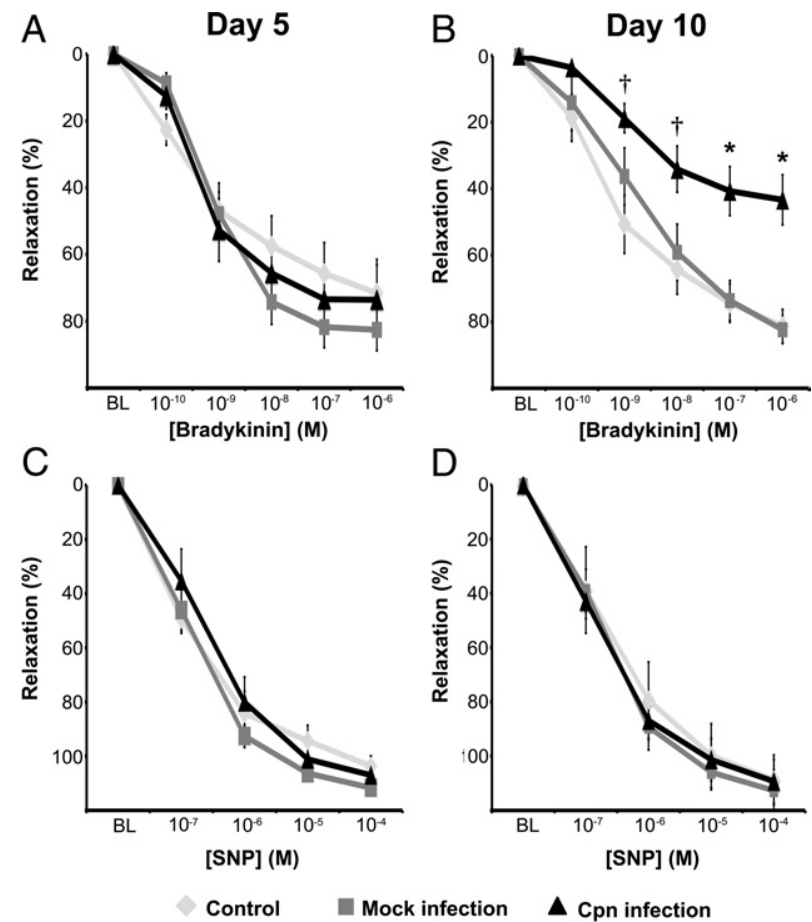

Figure 4. Relaxation responses of infected isolated vascular rings. Vacular preparations were relaxed to $10^{-10}-10^{-6}$ bradykinin day 5 postinfection (A) and day 10 postinfection (B) or $10^{-7}-10^{-4}$ sodium nitroprusside day 5 postinfection (C) and day 10 postinfection (D) after precontraction with 30 $\mathrm{nmol} / \mathrm{L}$ u46619. All values are mean $\pm \mathrm{SEM}, n=7$ to $11 .{ }^{*} P<0.05$ versus control and mock infection; ${ }^{\dagger} P<0.05$ versus control. 
A

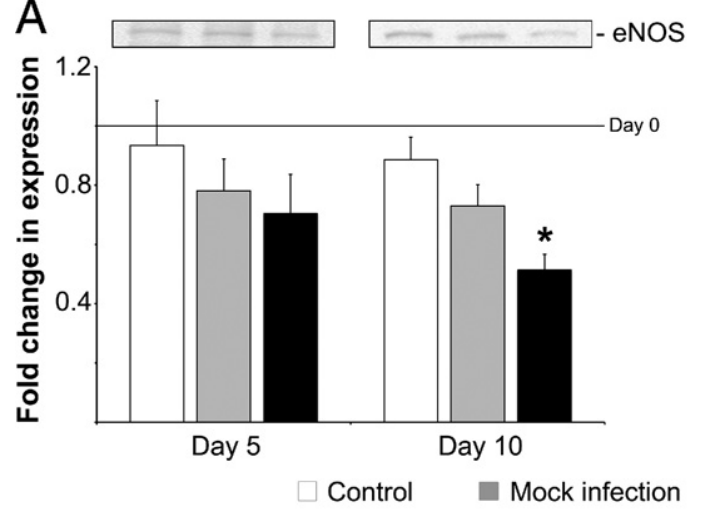

C

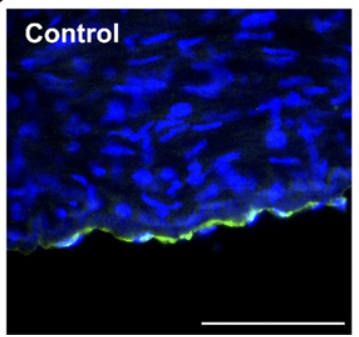

B

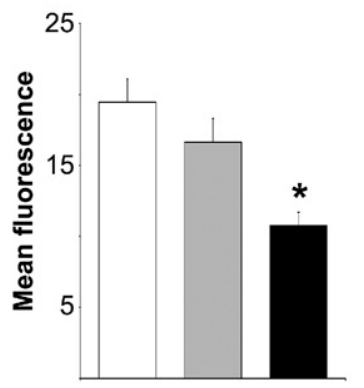

Cpn infection

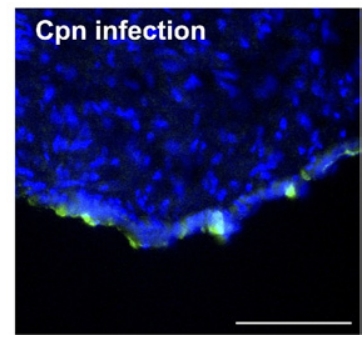

Figure 5. eNOS expression in infected isolated coronary arteries. A: Western blot analysis of whole tissue lysates at day 5 and day 10 postinfection. Representative Western blots are displayed above graph. Expression levels were represented as fold change over day 0 tissue \pm SEM, $n=4$ to 6 . Representative IHC staining (B) quantification and $(\mathbf{C})$ images of vessels collected at day 10 postinfection. Fluorescence intensity $(\mathbf{B})$ is represented as mean \pm SEM, $n=4$. Hoescht, blue; eNOS, green. Scale bar $=100$ $\mu \mathrm{m} .{ }^{*} P<0.05$ versus control and mock infection.
Conditioned Medium Does Not Alter eNOS and p22phox Expression in HUVECs

Both eNOS and p22phox protein expression were evaluated in HUVECs treated with conditioned medium from days $4,6,8$, and 10 postinfection via Western blot. Expression of both eNOS and p22phox in HUVECs treated with conditioned medium from all four time points were not significantly different between treatments (data not shown). cHSP60 and hHSP60 levels within conditioned medium from the different treatments were analyzed by Western blot. All conditioned medium displayed cHSP60 and hHSP60 levels below the bottom level of our standard curve of $0.01 \mu \mathrm{g}$ of protein, equivalent to 0.08 $\mu \mathrm{g} / \mathrm{mL}$ from the nonconcentrated conditioned medium (data not shown). cHSP60 could not be detected in culture medium from control and noninfected coronary vessels.

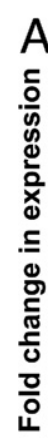

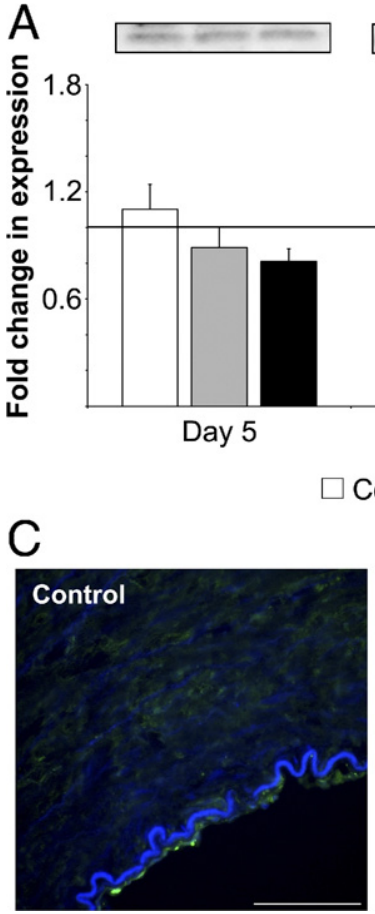

p22phox

B

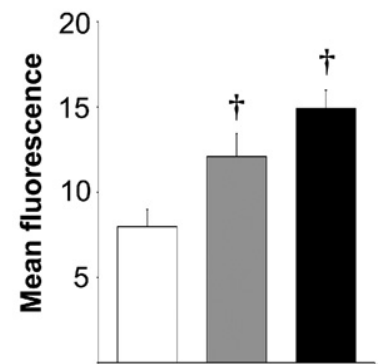

Cpn infection

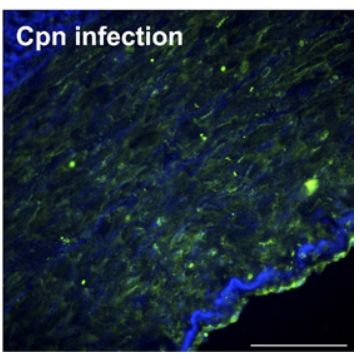

Figure 6. p22phox expression in infected isolated coronary arteries. A: Western blot analysis of whole tissue lysates at day 5 and day 10 postinfection. Representative Western blots are displayed above graph. Expression levels were represented as fold change over day 0 tissue \pm SEM, $n=4$ to 6 . Representative IHC staining (B) quantification and $(\mathbf{C})$ images of vessels collected at day 10 postinfection. Fluorescence intensity $(\mathbf{B})$ is represented as mean \pm SEM, $n=4$. Hoescht, blue; p22phox, green. Scale bar $=100$ $\mu \mathrm{m} .{ }^{\dagger} P<0.05$ versus control. 

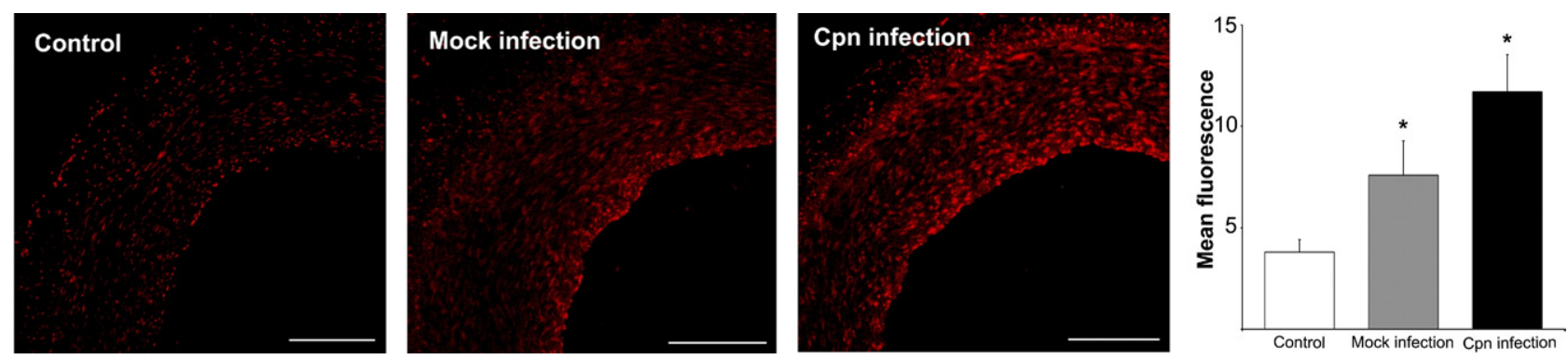

Figure 7. Superoxide detection by dihydroethidium staining in infected isolated coronary arteries. Representative staining of control, mock-infected, and $C p n$-infected vessels was collected at day 10 postinfection. Fluorescence intensity (right panel) is represented as mean \pm SEM, $n=4 ;{ }^{*} P<0.05$ versus control.

\section{Discussion}

We hypothesized that C. pneumoniae infection may participate in phenotype switching in VSMCs and as such may interfere with the ability of these cells to properly mediate the contractile and relaxation responses within an arterial environment. Our findings clearly demonstrate that $C$. pneumoniae infection leads to an impairment of both receptor (u46619) and depolarization (KCl)-mediated contractile responses (Figure 1). The involvement of the L-type $\mathrm{Ca}^{2+}$ channel and IP3R in this impairment was evaluated in the present study. In VSMCs, the L-type $\mathrm{Ca}^{2+}$ channel is the most important ion channel for the influx of extracellular $\mathrm{Ca}^{2+31}$ leading to downstream activation of myosin light chain kinase (MLCK). The channel can be opened directly through membrane depolarization by high concentrations of $\mathrm{K}^{+}$or indirectly through some agonists. ${ }^{32}$ The contractile responses elicited by u46619 are also dependent significantly on activation of the L-type $\mathrm{Ca}^{2+}$ channel. Inhibition of the L-type $\mathrm{Ca}^{2+}$ channel resulted in a $50 \%$ reduction of maximal force production generated by u46619 in porcine coronary arteries. ${ }^{33}$ In the present study, expression of the L-type $\mathrm{Ca}^{2+}$ channel was down-regulated at both day points when compared with control and mock-infected vessels (Figure 3, A and B). This is similar to the contractile changes exhibited in response to $\mathrm{KCl}$ and $\mathrm{u} 46619$. IP3R, found on the sarcoplasmic reticulum (SR), is also involved in MLCK activation through the release of SR $\mathrm{Ca}^{2+}$ stores in response to agonist-mediated G-proteincoupled signaling. ${ }^{32}$ IP3R expression in infected coronary vessels remained unaffected at day 5 postinfection but was significantly down-regulated at day 10 postinfection (Figure 3, C and D). Because a decrease in contraction at day 5 postinfection was observed without a change in IP3R expression. and because a decrease in IP3R expression at day 10 postinfection did not result in a further reduction in contractile performance of infected tissue, the contribution of IP3R in the defects exhibited in response to $\mathrm{u} 46619$ is questionable. The most likely mechanism for the alterations in contractile responses to both high $\mathrm{K}^{+}$and $\mathrm{u} 46619$ is through the reduction in L-type $\mathrm{Ca}^{2+}$ channel expression induced by C. pneumoniae infection. A decrease in the L-type $\mathrm{Ca}$ channel expression occurring in synthetic VSMCs is accompanied with an attenuation in smooth muscle actin and smooth muscle myosin heavy chain expression, two key components of the contractile apparatus. In the current study, although not significant, a decreasing trend in smooth muscle actin and smooth muscle myosin heavy chain expression in C. pneumoniae-infected vessels can be observed. Considering the mixed population of VSMCs (contractile and synthetic), it is possible the
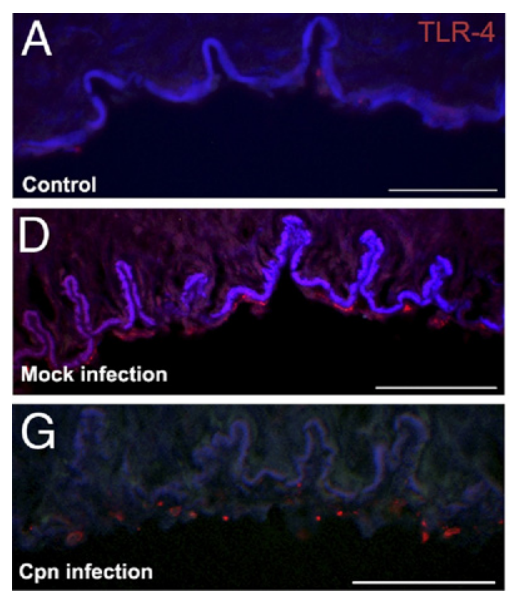
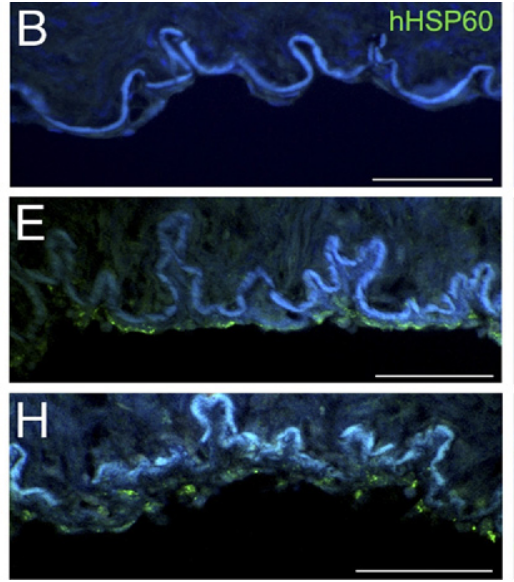
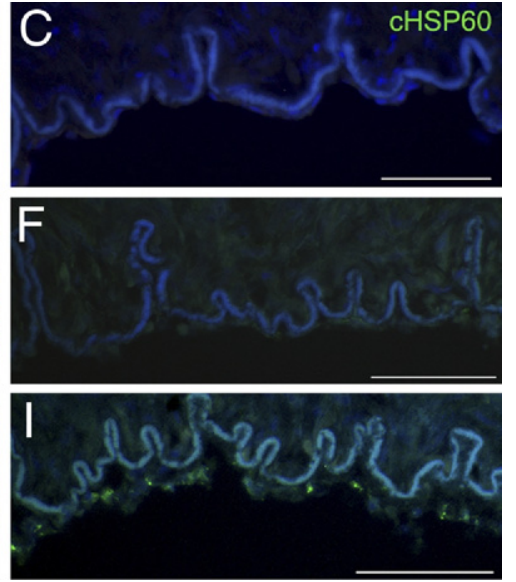

Figure 8. TLR- 4 , hHSP60, and cHSP60 localization within infected isolated coronary arteries. Representative staining of control (A-C), mock-infected (D-F), and Cpn-infected (G-I) vessels collected at day 10 postinfection. All panels: Hoescht, blue. A, D, G: TLR-4, red. B, E, H: hHSP60, green. C, F, I: cHSP60, green. Scale $\mathrm{bar}=100 \mu \mathrm{m}$ 
abundant expression of these markers in the contractile VSMC population may be dampening the decreases in expression within the synthetic VSMC population, thus contributing to the overall expression observed within whole vessel lysates. As such, the potential contribution of both these contractile proteins in the altered contractile responses should not be completely dismissed.

Other mechanisms beyond simple changes in contractile machinery expression could be considered for the observed contractile responses. Structural changes in C. pneumoniae-infected vessels have previously been noted using this same model system. ${ }^{27}$ Synthetic VSMCs demonstrate an increase in extracellular matrix production. ${ }^{25}$ As such, an increase in medial thickening via VSMCs proliferation could, in theory, confer changes in vessel stiffness through increased collagen production. However, it is important to note that these potential alterations in vessel stiffness would likely occur at later time points as medial thickening only starts to occur from day 6 postinfection onward. ${ }^{27}$ It is, then, unlikely that the changes in vascular contractile function observed at day 5 postinfection are due to this phenomenon. In addition, the attenuation in contractile responses in C. pneumoniae-infected vessels is not further aggravated at day 10 postinfection, which suggests vessel stiffness is likely not a major contributor.

C. pneumoniae infection also induced a significant attenuation in bradykinin-induced relaxation of precontracted tissue (Figure 4B). Bradykinin elicits relaxation of vascular tissue through an endothelial-dependent mechanism. ${ }^{34}$ The impairment in vasorelaxation was limited to the endothelial layer in the present study, as C. pneumoniae infection did not affect relaxation induced by the nitric oxide (NO) donor sodium nitroprusside. C. pneumoniae is known to induce endothelial damage. ${ }^{21}$ However in this model, C. pneumoniae infection did not induce endothelial cell death. ${ }^{27}$ Therefore, the attenuated relaxation response is more likely due to a cellular mechanism and not a deterioration of the endothelial layer.

Alterations to the (eNOS)/NO pathway, leading to a reduction in NO bioavailability, represent the most important mechanism for impaired endothelial-dependant relaxation. ${ }^{35}$ The relaxation responses to bradykinin in porcine coronary arteries is endothelial NO dependent. ${ }^{36}$ Bouwman et al have previously noted that $C$. pneumoniae infection in HUVECs results in a decrease in eNOS and downstream cGMP down-regulation. ${ }^{18}$ Our data further support this mechanism, as C. pneumoniae infection is also capable of inducing a decrease in eNOS expression in this organ culture system. However, it is unclear whether these changes occur directly as a result of live $C$. pneumoniae within the endothelial layer. As previously described with this model, there is a significant migration of $C$. pneumoniae from the endothelium into the medial area of coronary arteries from day 6 postinfection and onward. It is difficult to determine whether the $C$. pneumoniae population remaining in the endothelium at day 9 to 10 postinfection is large enough to alone account for these changes in eNOS expression. Alternatively, secretory factors released by the host cells or the bacteria itself within the neighboring medial layer may play a role in the changes observed. cHSP60 represents an potential candidate for such a role; it is highly expressed in C. pneumoniae infected coronary arteries, ${ }^{27}$ and Chen et al established its ability to down-regulate eNOS expression and activity in human coronary artery endothelial cells and porcine coronary arteries via TLR-4 signaling. ${ }^{19}$ We have demonstrated that $\mathrm{CHSP60}$ expression is localized with TLR-4 along the endothelial layer in C. pneumoniainfected vessels (Figure 8, G and I). However, conditioned medium retrieved from $C$. pneumoniae-infected vessels was unable elicit changes in eNOS and cHSP60 levels were well below the concentrations used by Chen et al. ${ }^{19}$ These data would suggest that CHSP60 secreted from infected VSMCs and/or endothelial cells is quickly binding TLR-4 along the endothelium and eliciting changes in eNOS.

In addition to a reduction in NO production, NO breakdown by superoxide radicals represents another mechanism for the $C$. pneumoniae-derived impairment in endothelial-dependent relaxation. Dihydroethidium staining of coronary vessels revealed an increase in superoxide production in both mock-infected and $C$. pneumoniae-infected vessels (Figure 7). Among the many sources of reactive oxygen species, NADPH oxidase is a major producer of superoxide within the vessel wall. ${ }^{37}$ p22phox, a major component of NAPDH oxidase, is expressed in ECs, VSMCs, adventitial fibroblasts and macrophages within human atherosclerotic plaques. ${ }^{38}$ Expression of p22phox expression was upregulated in both mock-infected and $C$. pneumoniaeinfected vessels at day 10 postinfection (Figure 6B). Furthermore, its expression was identified in both endothelial and medial layers (Figure 6C). The ability for the heat-inactivated $C$. pneumoniae to also elicit this response suggests that this effect is lipopolysaccharide mediated. Wick et al described that in vivo lipopolysaccharide administration increases hHSP6O expression on the surface of vascular endothelial cells. ${ }^{39}$ Our results indicate that $C$. pneumoniae and mock infection result in an increase in hHSP6O expression along the endothelium. In addition to down-regulating eNOS, Chen et al also described the ability of cHSP60 to up-regulate oxidative stress machinery including p22phox. Because of a conserved sequence homology for HSP60 between species, it is possible that hHSP60 may also induce similar downstream cellular changes through TLR-4 signaling.

The maintenance of isolated coronary vessels in a tissue culture environment for up to 10 days does not significantly alter the functional integrity of these arteries. ${ }^{26}$ Thus, the alterations observed in the present study are directly dependent on exposure to $C$. pneumoniae and/or C. pneumoniae components. The findings of the current study clearly demonstrate that $C$. pneumoniae infection is able to directly induce a significant impairment to the functional capacity of arteries. These defects are observed before (at 5 days) and after (10 days) significant thickening (hypertrophy) of the aorta is observed. ${ }^{26}$ These changes may have physiological consequences with regard to regulating normal blood flow, and may play an important role in 
the pathophysiology of hypertension, restenosis, and atherosclerosis.

\section{References}

1. Saikku P, Leinonen M, Mattila K, Ekman MR, Nieminen MS, Makela $\mathrm{PH}$, Huttunen JK, Valtonen V: Serological evidence of an association of a novel Chlamydia. TWAR, with chronic coronary heart disease and acute myocardial infarction, Lancet 1988, 2:983-986

2. Elkind MS, Tondella ML, Feikin DR, Fields BS, Homma S, Di Tullio MR: Seropositivity to Chlamydia pneumoniae is associated with risk of first ischemic stroke. Stroke 2006, 37:790-795

3. Danesh J, Whincup P, Lewington S, Walker M, Lennon L, Thomson A, Wong YK, Zhou X, Ward M: Chlamydia pneumoniae IgA titres and coronary heart disease; prospective study and meta-analysis. Eur Heart J 2002, 23:371-375

4. Siscovick DS, Schwartz SM, Corey L, Grayston JT, Ashley R, Wang SP, Psaty BM, Tracy RP, Kuller LH, Kronmal RA: Chlamydia pneumoniae, herpes simplex virus type 1 , and cytomegalovirus and incident myocardial infarction and coronary heart disease death in older adults: the Cardiovascular Health Study. Circulation 2000, 102:23352340

5. Shor A, Kuo CC, Patton DL: Detection of Chlamydia pneumoniae in coronary arterial fatty streaks and atheromatous plaques. S Afr Med $J$ 1992, 82:158-161

6. Ramirez JA: Isolation of Chlamydia pneumoniae from the coronary artery of a patient with coronary atherosclerosis. The Chlamydia pneumoniae/Atherosclerosis Study Group. Ann Intern Med 1996, 125: 979-982

7. Jackson LA, Campbell LA, Kuo CC, Rodriguez DI, Lee A, Grayston $\mathrm{JT}$ : Isolation of Chlamydia pneumoniae from a carotid endarterectomy specimen. J Infect Dis 1997, 176:292-295

8. Grayston JT, Kuo CC, Coulson AS, Campbell LA, Lawrence RD, Lee MJ, Strandness ED, Wang SP: Chlamydia pneumoniae (TWAR) in atherosclerosis of the carotid artery. Circulation 1995, 92:3397-3400

9. Maass M, Gieffers J, Solbach W: Atherogenetically relevant cells support continuous growth of Chlamydia pneumoniae. Herz 2000, 25:68-72

10. Godzik KL, O'Brien ER, Wang SK, Kuo CC: In vitro susceptibility of human vascular wall cells to infection with Chlamydia pneumoniae. J Clin Microbiol 1995, 33:2411-2414

11. Gaydos CA, Summersgill JT, Sahney NN, Ramirez JA, Quinn TC: Replication of Chlamydia pneumoniae in vitro in human macrophages, endothelial cells, and aortic artery smooth muscle cells. Infect Immun 1996, 64:1614-1620

12. Davignon J, Ganz P: Role of endothelial dysfunction in atherosclerosis. Circulation 2004, 109:|II27-III32

13. Prasad A, Zhu J, Halcox JP, Waclawiw MA, Epstein SE, Quyyumi AA: Predisposition to atherosclerosis by infections: role of endothelial dysfunction. Circulation 2002, 106:184-190

14. Liuba P, Karnani P, Pesonen E, Paakkari I, Forslid A, Johansson L, Persson K, Wadstrom T, Laurini R: Endothelial dysfunction after repeated Chlamydia pneumoniae infection in apolipoprotein E-knockout mice. Circulation 2000, 102:1039-1044

15. Liuba P, Karnani P, Pesonen E, Paakkari I, Persson K, Forslid A: Effects of bradykinin on aortic endothelial function in apoe-knockout mice with chronic Chlamydia pneumoniae infection. Circ J 2007, 71:1480-1484

16. Liuba P, Pesonen E, Forslid A, Paakkari I, Kornerup-Hansen A, Kovanen P, Pentikainen M, Persson K, Ostergard G: Protective effects of simvastatin on coronary artery function in swine with acute infection. Atherosclerosis 2006, 186:331-336

17. Liuba P, Pesonen E, Paakkari I, Batra S, Forslid A, Kovanen P, Pentikainen M, Persson K, Sandstrom S: Acute Chlamydia pneumoniae infection causes coronary endothelial dysfunction in pigs. Atherosclerosis 2003, 167:215-222

18. Bouwman JJ, Visseren FL, Bevers LM, van der Vlist WE, Bouter KP, Diepersloot RJ: Azithromycin reduces Chlamydia pneumoniae-induced attenuation of eNOS and cGMP production by endothelial cells. Eur J Clin Invest 2005, 35:573-582
19. Chen C, Chai H, Wang X, Lin PH, Yao Q: Chlamydia heat shock protein 60 decreases expression of endothelial nitric oxide synthase in human and porcine coronary artery endothelial cells. Cardiovasc Res 2009, 83:768-777

20. Shi Y, Tokunaga O: Chlamydia pneumoniae (C. pneumoniae) infection upregulates atherosclerosis-related gene expression in human umbilical vein endothelial cells (HUVECs). Atherosclerosis 2004, 177: 245-253

21. Hirono S, Dibrov E, Hurtado C, Kostenuk A, Ducas R, Pierce GN: Chlamydia pneumoniae stimulates proliferation of vascular smooth muscle cells through induction of endogenous heat shock protein 60 . Circ Res 2003, 93:710-716

22. Kol A, Bourcier T, Lichtman AH, Libby P: Chlamydial and human heat shock protein 60s activate human vascular endothelium, smooth muscle cells, and macrophages. J Clin Invest 1999, 103:571-577

23. Halcox JP, Deanfield J, Shamaei-Tousi A, Henderson B, Steptoe A, Coates AR, Singhal A, Lucas A: Circulating human heat shock protein 60 in the blood of healthy teenagers: a novel determinant of endothelial dysfunction and early vascular injury?. Arterioscler Thromb Vasc Biol 2005, 25:e141-e142

24. Gollasch M, Haase H, Ried C, Lindschau C, Morano I, Luft FC, Haller $\mathrm{H}$ : L-type calcium channel expression depends on the differentiated state of vascular smooth muscle cells. FASEB J 1998, 12:593-601

25. Rzucidlo EM, Martin KA, Powell RJ: Regulation of vascular smooth muscle cell differentiation. J Vasc Surg 2007, 45 Suppl A:A25-A32

26. Sasu S, LaVerda D, Qureshi N, Golenbock DT, Beasley D: Chlamydia pneumoniae and chlamydial heat shock protein 60 stimulate proliferation of human vascular smooth muscle cells via toll-like receptor 4 and p44/p42 mitogen-activated protein kinase activation. Circ Res 2001, 89:244-250

27. Deniset JF, Cheung PK, Dibrov E, Lee K, Steigerwald S, Pierce GN: Chlamydophila pneumoniae infection leads to smooth muscle cell proliferation and thickening in the coronary artery without contributions from a host immune response. Am J Pathol 2010, 176:10281037

28. Kuo CC GJ: A sensitive cell line. HL cells, for isolation and propagation of Chlamydia pneumoniae strain TWAR. J Infect Dis 1990, 162: 755-758

29. Furness G, Graham DM, Reeve P: The titration of trachoma and inclusion blennorrhoea viruses in cell cultures. J Gen Microbiol 1960, 23:613-619

30. Zanetti M, d'Uscio LV, Peterson TE, Katusic ZS, O'Brien T: Analysis of superoxide anion production in tissue. Methods Mol Med 2005, 108: 65-72

31. Orallo F: Regulation of cytosolic calcium levels in vascular smooth muscle. Pharmacol Ther 1996, 69:153-171

32. Somlyo AP, Somlyo AV: Signal transduction and regulation in smooth muscle. Nature 1994, 372:231-236

33. Nobe K, Paul RJ: Distinct pathways of $\mathrm{Ca}(2+)$ sensitization in porcine coronary artery: effects of Rho-related kinase and protein kinase $\mathrm{C}$ inhibition on force and intracellular $\mathrm{Ca}(2+)$. Circ Res 2001, 88:12831290

34. Hornig B, Drexler H: Endothelial function and bradykinin in humans. Drugs 1997, 54 Suppl 5:42-47

35. Kawashima S, Yokoyama M: Dysfunction of endothelial nitric oxide synthase and atherosclerosis. Arterioscler Thromb Vasc Biol 2004, 24:998-1005

36. Danser AH, Tom B, de Vries R, Saxena PR: L-NAME-resistant bradykinin-induced relaxation in porcine coronary arteries is NOdependent: effect of ACE inhibition. Br J Pharmacol 2000, 131:195202

37. Griendling KK, Sorescu D, Ushio-Fukai M: NAD(P)H oxidase: role in cardiovascular biology and disease. Circ Res 2000, 86:494-501

38. Azumi H, Inoue N, Takeshita S, Rikitake $Y$, Kawashima S, Hayashi $Y$, Itoh $\mathrm{H}$, Yokoyama M: Expression of NADH/NADPH oxidase p22phox in human coronary arteries. Circulation 1999, 100:1494-1498

39. Wick MC, Mayerl C, Backovic A, van der Zee R, Jaschke W, Dietrich $H$, Wick G: In vivo imaging of the effect of LPS on arterial endothelial cells: molecular imaging of heat shock protein 60 expression. Cell Stress Chaperones 2008, 13:275-285 